\title{
Manipulación de objetos y comunicación en crías gorila cautivas. Nuevos comentarios para un debate inacabable
}

\author{
Adolfo Perinat \\ Universidad Autónoma de Barcelona
}


En el número 38 de Estudios de Psicología (verano 1989) Juan Carlos Gómez ha publicado un artículo, "La comunicación y la manipulación de objetos en crías de gorila» en que toma como referencia el publicado por mi, en colaboración con A. Dalmau, en la misma revista. Números 33-34, y con el título «La comunicación entre pequeños gorilas criados en cautividad y sus cuidadoras». El trabajo de J. C. Gómez es un comentario crítico al nuestro.

Vaya por delante que me congratulo de haber merecido la atención de un especialista en las cuestiones tratadas en nuestro artículo original y de entablar una sana polémica sobre el tema que nos concierne. Suele ser, de todos modos, una honorable costumbre que el discrepante envíe al autor de referencia su texto, previamente a su publicación, a fin de que éste tenga la oportunidad de insertar su respuesta y comentarios dentro del mismo ejemplar en que aquel expone sus puntos de vista. Diversas circunstancias han concurrido que ejerza mi derecho de réplica con un retraso que aquí trato de subsanar.

\section{LA MANIPULACION DE OBJETOS EN NUESTROS GORILAS Y EN LOS PRIMATES}

Nuestro trabajo nos llevó a varias conclusiones con respecto a la manipulación de objetos (humanos) en los gorilas y, por encima de este comportamiento, a una interpretación global del comportamiento manipulativo en los primates. La primera fue que «nuestros gorilas están escasamente motivados para manipular objetos» (subrayado añadido ahora). Una segunda conclusión fue que la comparación entre la interacción de las crías gorila y de los niños con adultos humanos en juegos manipulativos deja al descubierto profundas diferencias. Tercera, y como consecuencia de lo anterior, que la manipulación primate exige una interpretación mas ecológica y menos antropocéntrica.

J. C. Gómez asegura que su gorila, Muni, «mostró asiduamente manipulaciones sencillas y complejas, tanto en situaciones motivadas extrínsecamente como intrínsecamente». De lo cual me congratulo sinceramente. Donde nosotros apenas logramos éxito, nuestro colega lo ha conseguido plenamente. Aparte de avanzar algunas conjeturas acerca del por qué de tales diferencias quiero argumentar que, aunque sus datos discrepan de los míos, no rebaten (o «falsifican» como reza el barbarismo popperiano) mis conclusiones.

Comenzaré por el tema de las «reacciones circulares secundarias». Si por algo se caracterizó el comportamiento observado en nuestros pequeños gorilas fue por la abundancia de las mismas. Ahora bien, en mi opinión, está fuera de lugar introducir la noción de motivación intrínseca en relación con estos comportamientos de una funcionalidad biológica muy primitiva, de una intencionalidad tan embrionaria. La motivación intrínseca se aplica actividades intencionadas que incluyen secuencias de esquemas; el plan se confunde con la propia actividad, o en otros términos, no hay disyunción entre lo que se hace y lo que se pretende conseguir; hay una dimensión cognitiva, de procesamiento de información (o standard) subyacente, etc. Las ideas de White (1959), Hunt (1965), Nuttin (1980), Eckblad (1981) en las que me inspiro al asignar estas características a la motivación intrínseca sugieren que es improcedente adscribir este estado motivacional al niño (o gorila) que ejecuta reacciones circulares primarias o secundarias. 
No voy a insistir más en este punto. Creo que es suficiente para disipar la contradicción que graciosamente me atribuye J. C. Gómez en la pág. 115 de su escrito, contradicción que el minimiza al reconocer (con toda razón, esta vez) que el núcleo de mi argumentación se centra en las reacciones circulares terciarias.

Sin duda que aquí el comportamiento de nuestros gorilas y el de Muni son claramente distintos. No me duelen prendas aceptar que Muni efectúa reacciones circulares terciarias mientras que nuestros gorilas apenas las realizaron. Pero está lejos de mi intención y de la información científica que poseo negar, por decreto, que se den en los gorilas o en los demas antropoides (incluso monos inferiores). En el libro de C. Hayes, The Ape in our House (1951) hay unas preciosas fotografías del chimpancé Viki en plena forma manipulativa. La cuestión no es, pues, de "all or nothing" sino de frecuencia, situaciones en que se dan y, sobre todo, de su significado funcional dentro del marco de desarrollo gorila. Nosotros registramos tres y muy rudimentarias (exceptuando la de Kena intentando recomponer el teléfono); J. C. Gómez las ha registrado con mayor frecuencia y son de mayor complejidad. Los gorilas en que han sido observadas han sido criados por humanos y están en contacto asiduo con ellos. ¿Ocurre otro tanto con los gorilas observados en naturaleza? La lectura atenta de D. Fossey, Gorilas in the Mist (1983) es particularmente parca en la descripción de manipulaciones complejas (reacciones circulares terciarias). Una notable excepción son los episodios de juego de Puck, con los anteojos y con el Geographical Magazine (págs. 80-81). A. Dalmau que pasó dos meses observando a los mismos gorilas de la $\mathrm{D}$. Fossey confirmó que el interés que manifestaban por los objetos de los observadores humanos era fugaz y no daba pie a exploraciones manipulativas. Las investigaciones sobre primates salvajes no abundan en descripciones de manipulaciones de objetos que pueblan el entorno natural y que entran en la categoría de reacciones circulares terciarias. ¿Es porque son relativamente escasas o bien porque no han sido debidamente registradas? (Esto último quizás haya que achacarlo a que se trata de una conducta no relevante para los primatólogos ajenos a las ideas de Piaget; lo contrario de lo que ocurre con la conducta instrumental que ha sido tradicionalmente "la estrella»). Una dificultad añadida es que la noción de manipulación es vasta y difusa y por eso el argumento de autoridad que aduce J. C. G. ("la capacidad de los antropoides para efectuar manipulaciones instrumentales se encuentra suficientemente documentada en la literatura» pág. 116) no tiene mucha fuerza. Los autores llaman manipulación a una panoplia de actividades de lo más diverso: desde asir algo con la mano hasta acciones sobre objetos que implican transformaciones de los mismos (tipo la del chimpacé de Köhler o las reacciones circulares terciarias que aquí estámos discutiendo).

De todas maneras, estas últimas se dan pero insisto en que la mayoría de las repertoriadas se han observado en cautividad y que esto no es una mera secuela de que aquí hay más oportunidad de registrarlas. En la naturaleza existen palitos, raices, piedras, cortezas, etc. etc. que darían pábulo a la actividad exploratorio-transformadora manual. ¿Serían, pues, extrapolables las frecuencias de reacciones circulares terciarias que atribuye J. C. Gómez a su gorila a los gorilas salvajes? En otros términos, ¿es esta una conducta «natural» en los gorilas? ¿O es más bien una conducta «accesible» a sus capacidades en determinadas circunstancias? 
La noción de «comportamiento natural» es sumamente espinosa. Estrictamente hablando significaría un comportamiento típico, propio de una especie (species specific) tal y como es detectado en la naturaleza y registrado en los etogramas. Por razones obvias, es practicamente imposible decidir si las reacciones circulares terciarias son un comportamiento natural. Pero, aunque no fuere natural, strictu sensu, tampoco hay que concluir que sea de esos comportamientos artificiales, extravagantes, anómalos o, en el extremo, «patológicos» que a veces se dan en los animales cautivos (por ejemplo, los de circo). En los animales superiores existe la capacidad de incorporar nuevos comportamientos a su repertorio. Los primeros exhiben un conjunto de esquemas manuales propios: asir, empujar, apantallarse el rostro, etc. Son «naturales» en ellos como es asimismo "natural» en los antropoides la capacidad de ajustarlos a objetos nuevos e incluso de «construir» alguno especial para determinadas manipulaciones: abrir cajas, manejar instrumentos (llaves, tornillos...) encajar/desencajar, etc. etc. Son esquemas aprendidos, que si bien no son «naturales» por cuanto no forman parte de los comportamientos observables en la naturaleza, si lo son en cuanto que aparecen como una extensión «natural» de capacidades manuales existentes. En otros términos, son propios en la medida que son fruto de una capacidad de acomodación que es propia de su nivel evolutivo. La noción piagetiana de aprendizaje (Ver La naissance de l'intelligence chez l'enfant, cap. V, 2) entra aquí de pleno.

No estará de más hacer notar dos circunstancias que concurren en la vida y entorno de los gorilas criados por humanos que ayudan a explicar la emergencia de estas conductas (o, si se prefiere, su frecuencia mayor). La primera es la propia existencia de objetos (culturales) como entes distintos de las «cosas» que pueblan el entorno natural de la selva donde ha evolucionado la especie. Los objetos humanos están especialmente diseñados para superponerse, despiezarse, combinarse, encajarse/desencajarse, etc. Constituyen una fuente de "affordances" combinatorio-manipulatorias sumamente rica. Pero esto es solo parte de la historia pues el entorno natural daría pie a inventar parecidos efectos con palitos, cáscaras de frutas, cortezas, hojas, etc. Lo que falta - y esto es lo decisivo- son los «marcos» instrumental, modelador y de feedback (en expresión de K. Kaye, 1982) que crean los adultos en su recurso constante, utilitario y también lúdico, a los objetos. Como consecuencia de todo ello los antropoides ni se entretienen demasiado en efectuar exploraciones y combinaciones ni, menos aún, «descontextualizan» o «desvirtúan» el uso natural de sus hojas, raices, palitos, etc. haciendo "como si» (juego simbólico). Las causas de ello son diversas pero se relacionan íntimamente entre sí. Fundamentalmente no existe entre adultos y crías una interacción lúdica con los objetos (ni siquiera existen demostraciones ni «enseñanza» directa de cómo efectuar determinadas manipulaciones, como la construcción de nidos). "C'est la faute à Piaget» de habernos presentado a un niño explorador solitario y ejecutante concentrado de sus manipulaciones, combinaciones, como si la realidad no fuera que la fuente de inspiración de sus cachivacheos está en los adultos. No deja de ser llamativo que las madres chimpancé no cooperan en los juegos manipulativos a que eventualmente se entregan sus crías. Por más motivado que estuviere un niño (¿un antropoide?) para manipular, su actividad se apagaría si no fuera por que vive en un entorno social «instrumental» que crea relaciones muy peculiares en torno a la manipulación instrumental, primero lúdica y luego transformadora. 
Los gorilas criados en un ambiente enriquecido y en frecuente contacto con los humanos, como es el caso que describe J. C. Gómez, progresan mucho más que otros en la manipulación y la interacción. Los nuestros, aunque muy familiares con sus cuidadoras, no tuvieron, en este aspecto, un trato particularmente rico y estimulante. Ademas eran una pareja, es decir, no establecían una relación exclusiva y privilegiada con los humanos. Sospecho que esto último tiene más importancia de la que a primera vista se podría suponer pero necesitaría de otros estudios comparativos para demostrarlo concluyentemente.

En definitiva, ghemos de concluir que los pequeños gorilas o los chimpancés a quienes descubrimos manipulando entretenidamente junto a los humanos son equiparables a los niños en cuanto a este comportamiento se refiere? Dos comportamientos materialmente idénticos en cuanto a su «forma» puede que no tengan el mismo significado funcional en dos especies distintas en la perspectiva de la ontogénesis del repertorio conductual de cada una de ellas. En los gorilas, a diferencia de los niños, las manipulaciones complejas no constituyen un eslabón intermedio de la cadena de comportamientos transformadores del entorno, cadena que se prolonga en las coordinaciones complejas inherentes a la acción instrumental, en la creación recursiva de útiles, en la comunicación convencional y simbólica, en la cooperación tecnológica que ha hecho nacer la cultura humana. Es un añadido casual, un paso "perdido" dentro de una avenida evolutiva que para ellos ya se ha cerrado definitivamente. A menos que se pretenda que en la manipulación de primates criados por humanos pueda estar en el origen de un «salto» evolutivo que los aproxime o iguale a los humanos en un futuro indeterminado. Esto es ciencia ficción. En cambio, en el marco de las funciones de supervivencia básicas, los esquemas manuales de los primates guardan toda su virtualidad. Son estas las razones que justifican dos afirmaciones que no comparte mi crítico, a saber, que el recurso a las "cosas" está primordialmente orientado hacia la satisfacción de las necesidades primarias y que "la mano primate tiene una función casi totalmente subordinada a otras funciones más vitales como son alcanzar, transportar, asir, apantallarse el rostro, repeler, etc.». Aquí introduzco la noción de "campo psicológico»: conjunto de funciones que al nivel de evolución de una especie son adscribibles a determinadas estructuras o comportamientos. Un ejemplo muy claro es el «campo psicológico» del comportamiento sexual. El apareamiento es su denominador común, la función biológica de la reproducción también lo es; pero nadie negará que el sexo posee otras funciones incorporadas por la evolución en la especie humana que hacen de este comportamiento algo distintivo en ella, de un carácter sui generis y, por añadidura, inaccesible a otras especies inferiores.

\section{LA COMUNICACION DE LOS PEQUEÑOS GORILAS CON LOS HUMANOS}

No hay ninguna ambigüedad en mi escrito, contra lo que sugiere J. C. Gómez, acerca de que los gorilas tienen intenciones comunicativas y que, a su manera, se hacen entender de los humanos. Es seguro, con todo, que más de un movimiento o «forma postural» de los pequeños gorilas, dotada de intención comunicativa, nos sea inaccesible. Los humanos entendemos a los antropoides extrapolando a partir de la conducta intencionada o comunicativa propiamente 
humana. Y esta extrapolación es lícita porque nuestra percepción y nuestra mente están capacitadas para captar - a partir de la expresividad inherente al movimiento- estados de ánimo y estados motivacionales que se traducen en intenciones de hacer algo o de dirigirse al alter para influir en él. Esta es la intersubjetividad primaria, que tiene unos fundamentos psicobiológicos (Perinat, 1989) y que "funciona" bastante bien entre antropoides y humanos. No funciona omnímodamente ni tiene el mismo curso o alcance de humanos a antropoides que recíprocamente.

Pero no es este el aspecto que más me interesa comentar sino lo que J. C. G. denomina actos comunicativos «triangulares», esto es, aquellos que involucran el traspaso de objetos o su manipulación conjunta entre interlocutores. Comienzo por algunas presiones a la idea de «comunicación triangular». Los objetos pueden estar involucrados en las acciones comunicativas en grados diversos. Por ejemplo, pedir algo y acceder a la petición, poner en manos de un adulto determinado objeto para que éste coopere abriendo/cerrando, enroscando/desenroscando, etc. son actos comunicativos porque prestamos al demandante que hace el gesto de pedir o mostrar, la intención de conseguir del otro una determinada conducta; el primer actor posee una representación del otro (teoría de la mente) como de alguien a quien es accesible la acción expresiva con el objeto de por medio. Las obs. 10,11, 12 y 13 que cita J. C. G. en su artículo son de este tipo. El mero hecho de que exista este objeto sobre el que, en alguna manera, versa la comunicación es lo que al parecer justifica que él la llame «triangular». Pero compárense estas observaciones con las 16 y 17. Aunquue sigue en ellas interviniendo un objeto, la diferencia estriba en cómo los interlocutores definen su papel mutuo y el del objeto en el decurso de la interacción. En las situaciones descritas en las observaciones anteriores (recurso a la acción instrumental del interlocutor más diestro) el papel de éste y el tipo de acción son impuestas por el solicitante. Ahora, en el caso del "toma y daca" (Obs. 16) o en los formatos de acción conjunta (construcciones, seriaciones a duo) existe una sucesión de esquemas alternante que se despliegan dentro de un plan para «conseguir algo». No es necesario que ese algo esté anticipado con igual detalle por ambas mentes; puede ser «negociado» en el decurso de la interacción de tal modo que el mismo conjunto de objetos da pie a «efectos» diferentes. Ahora la actividad es libre (lúdica) y el objeto deviene el «pretexto» para mantener la interacción; ésta es tan comunicativa como ejecutiva. Este mantenimiento de la interacción ocupa un primer plano en las intenciones (motivación) subyacentes y el efecto conseguible con el objeto pasa a un segundo plano, incluso puede ser renegociado o improvisado. Como puede colegirse, estoy imponiendo la condición de que ambos interactantes estén imbuídos de una motivación intrínseca compartida. Se trata, además, de una típica instancia vygotskiana: se genera en la interacción un estado intermental que es trasvasado a cada una de las mentes participantes. Es plenamente un «shared understanding".

Las condiciones que acabo de enumerar hacen de este segundo tipo de interacciones algo bastante diferente y mucho más sofisticado que las primeras. $\mathrm{Si}$ aquellas prolongan los protoimperativos, éstas recuerdan los protodeclarativos. Dado que los antropoides no hacen este tipo de acciones comunicativas habría que observar muy finamente las «acciones conjuntas lúdicas» que los pequeños gorilas del Zoo de Madrid llevan a cabo (obs. 16 y 17 que describe J. C. G.) y compararlas con sumo cuidado con las de los niños. No basta la 
descripción verbal para esta comparación: la intensidad y la dirección de la atención en juego, la coordinación por parte de los actores de los esquemas de acción, el grado y la calidad de la motivación adscribible han de evaluarse y discutirse sobre escenas filmadas.

Entre estos dos extremos de «comunicaciones triangulares» existen otras intermedias. En más de un formato de acción conjunta el niño o el gorila pretende conseguir un efecto anticipable que se realizaría gracias a un conjunto de esquemas que aun no posee. Puede recurrir al adulto en diversas fases de su actividad. El adulto crea entonces lo que Bruner ha denominado una forma de "andamiaje» (scaffolding). Aquí las motivaciones puede que estén mezcladas; la atención del niño (o gorila) pasa de la actividad en si al objetivo que trata de conseguir y al adulto que le depara ayuda. Puede que el adulto esté más intrínsecamente motivado que la criatura a mantener la interacción en la línea que impone ésta pero puede también mantenerse simplemente abierto a cooperar eventualmente.

$$
* *
$$

Sentadas estas premisas, abordaré el tema de la intersubjetividad secundaria, una capacidad que J. C. G. llega a prestar a sus gorilas. Se trata de una disposición peculiar, una dimensión motivacional específica que se plasma en esas situaciones que $\mathrm{C}$. Trevarthen ha tipificado muy acertadamente de «sharing a task». La intersubjetividad secundaria es un marco motivacional que lleva a captar al otro como alguien con quien se puede concertar la acción (que deviene así «joint action»); pero no cualquier acción sino precisamente la que está mediada por los objetos o instrumentos. Es un estadio avanzado de la cooperación social la cual existe a muchos niveles de la filogenia con diversas manifestaciones y con intervención de mecanismos psicológicos de complejidad gradual. A lo que nos referimos cuando de la intersubjetividad secundaria se trata, es a ese tipo específico de cooperación que tiene su expresión definitiva en la transformación del entorno mediante el útil. La intersubjetividad secundaria brota de la primaria luego de un auténtico salto evolutivo y comporta la emergencia de nuevas capacidades. Enumero las siguientes. La primera es la de captar el movimiento que anima el útil como algo significativo y dotado de propósito. Lo cual no es una condición trivial puesto que supone la puesta a punto de una percepción innata que atribuye un significado a aquel movimiento, y que lo reviste del caracter de "plan». En otros términos, el gesto instrumental se convierte en una «affordance» gibsoniana. Y bien, mi tesis es que esto es algo específico de la especie bumana o sea que surge de novo en la transición de los antropoides a los homínidos. (En realidad la especie humana surge al mismo tiempo que las capacidades y procesos instrumentales y la transformación planeada, cooperativa, del entorno. Esta simultaneidad de procesos y de puesta a punto de niveles de organización psíquica superior es, por definición una autopoiesis. Maturana \& Varela, 1980). Nuestros gorilas demostraron ser absolutamente ciegos e indiferentes al gesto instrumental; refractarios a captar la intencionalidad inherente al mismo. Algo menos parece suceder con los gorilas del Zoo de Madrid. Pero «una golondrina no hace verano» $y$, una vez más, sostengo que la innegable destreza manual que poseen los antropoides no se despliega en un campo psicológico que expande su capacidad anatómiconerviosa a las funciones de transformación «tecnológica»del medio. La mani- 
pulación puede ser, en cambio, delicada y compleja cuando se trata de obtener alimento.

Una de las consecuencias de esta ineptitud a captar el «gesto manual» que esgrime el util es que la imitación de los casos (contados) que del mismo orden se dan en la naturaleza es muy pobre. Call y Alonso (1989) demuestran, luego de analizar cuidadosamente el comportamiento de unos chimpancés del Zoo de Barcelona con un termitero artificial, que la imitación - estrictamente hablando- es casi inexistente; que se puede hablar de «facilitación social» o de pistas-acerca-del-uso-del-instrumento (palitos para hurgar). Si esto es así, nada tiene de extraño que las instancias de innovación «cultural» entre los antropoides se hayan convertido en «anécdotas» extraordinarias. Que el uso «adecuado" de instrumentos no es capitalizado por los otros miembros del grupo, quedó inequívoca y sorprendentemente demostrado por J. Goodall a propósito de aquel chimpancé de Goombe que descubrió el «efecto tambor» (lo que le permitió subir en el ranking de la tropa): nadie tuvo «la ocurrencia» de reproducirlo.

La intersubjetividad secundaria es una conquista transcendental - an el sentidó genuino de esta palabra - porque a partir de ella se proyecta ese tipo especial de cooperación que es la que ba becho nacer la cultura bumana. Suele considerarse a la cultura como un apéndice de nuestra naturaleza (la clásica dicotomía naturaleza/cultura) pero, si reflexionamos un instante, no existe ninguna solución de continuidad entre una y otra (al menos filogenéticamente). Las disposiciones y motivaciones para crear/participar de la cultura son típicas de nuestra especie. La genialidad de $\mathrm{C}$. Trevarthen ha sido intuir este lazo profundo entre la intersubjetividad secundaria y la cultura que hemos creado los humanos al descubrir en los «sharing a task» más primerizos de los niños (9-10 meses) una disposición innata hacia formas de cooperación con y a través de los objetos. Aquí se sientan las bases de la cultura (el adjetivo «humana» es redundante, Tomasello 1988) y de la dimensión específicamente humana de la sociabilidad.

Los gorilas criados en ambiente enriquecido y en frecuente contacto con los humanos, como es el caso que describe J. C. G., progresan indudablemente en la manipulación e interacción. ¿Hasta dónde? Cuestión difícil de zanjar pues depende de muchas variables. Y es justamente la ausencia de una norma de desarrollo, de un conjunto de regularidades predecibles que se manifiestan en la emergencia jalonada de ciertas capacidades- las mismas para todos- lo que hace que la información que vamos acumulando sea tremendamente ambigüa. Es lo que ha ocurrido con el famoso tema del «lenguaje» de los antropoides.

Por tanto, más que buscar una respuesta directa a la expansión o a los límites de las capacidades antropoides o a la dirección que sigue su desarrollo (lo que J. C. G. enuncia como alternativa entre deficiencias versus divergencias), habría que plantear otra línea de indagación como: ¿qué conocimientos nuevos podemos extraer de estas situaciones acerca de las capacidades de la criatura humana para la comunicación y para el desarrollo cognitivo? ¿Qué papel juegan en ellas la manipulación compartida de los objetos? ¿Qué podemos intuir acerca de la transición filogenética antropoides-humanos? Porque de que los pequeños gorilas o los chimpancés llegan a manipular «de la misma manera" que los niños, no vamos a concluir que son "como» los niños. (Y soy muy consciente que las dos expresiones entrecomilladas son absolutamente 
refractarias a la precisión científica). Ya lo he comentado anteriormente y este es el núcleo de mi argumentación: decidir de la equivalencia de dos comportamientos en especies diferentes, por más que sean vecinas, es delicado. Y, cuando una de ellas es la humana, dado el arrollador despliegue psicológico que hemos tenido, toda comparación corre el riesgo de ser reduccionista.

Todo esto, aplicado a la intersubjetividad secundaria, nos lleva a sostener que el hecho de recurrir al adulto (humano) para que éste solucione un problema material, manipulativo, no es suficiente para asegurar que los gorilas acceden a ella. Las capacidades que el gorila pone aquí en juego podrían ser el umbral. Las situaciones «triangulares» no han de ser de mero intercambio sino de compartir una actividad. Tampoco la intersubjetividad secundaria es cuestión de "all or notbing». Pero lo que pretendo subrayar es que hay cualidades de la interacción, como la intensidad en la manera de participar, la atención concentrada simultáneamente en lo que se hace y en la coordinación simultáneamente en lo que se hace y en la coordinación/negociación de los esquemas que intervienen, la dimensión lúdica y otras que le adscribimos, -en base a lo que observamos y también a los marcos conceptuales que guían nuestra observación- que son elementos diferenciadores o definitorios. En razón luego de la visión prospectiva que tenemos del desarrollo humano y de la experiencia que nos depara la crianza, añadimos que el niño alimenta una disposición innata a considerar al adulto como un maestro de quien puede aprender no solo el uso «uril» de las cosas sino maneras y convenciones. Recíprocamente, el adulto está motivado para dedicarle su tiempo (que, por cierto, le escasea mucho más que a los gorilas de la naturaleza) para esta actividad modeladora y docente plegándose admirablemente a sus capacidades mentales incipientes.

Por añadidura, el que la intersubjetividad secundaria adquiera consistencia conceptual destacándose sobre un telón de fondo de comportamiento lúdico es teóricamente muy convincente. Permite encajar bastante bien las piezas del rompecabezas evolutivo de la motivación, manipulación de objetos, comunicación a dúo y el paso del juego movido al manipulativo. Todo esto lo señalo en mi artículo. Allí insisto en que la pieza clave podría ser la aparición del juego manipulativo «tranquilo» que se desgaja del «motor play» antropoide o mamífero como una conquista tardía de la filogenia favorecida por la heterocronía entre la coordinación perceptivo-manual, las capacidades de mutuo entendimiento (que hunden sus raíces en la intersubjetividad primaria) y la motricidad gruesa al ralenti.

\section{COMENTARIO FINAL: ¿PARA QUÉ LAS INVESTIGACIONES SOBRE LA CONDUCTA ANTROPOIDE?}

Mi preocupación al escribir esta respuesta a las objeciones del artículo de J. C. G. no ha sido poner en tela de juicio los datos de su investigación que constatan la existencia de comportamientos manipulativos y algunas modalidades comunicativas, las más simples, allí donde nosotros hemos constatado su ausencia casi total. Tampoco he querido matizar o refutar una por una interpretaciones que él me atribuye y que no he suscrito jamás (por ejemplo, las que me presta sobre la imitación o aquella de que los actos comunicativos en situaciones de juego son «excepcionales» con lo cual insinúa que hago 
explicaciones ad hoc...). El propósito (inconfesado) que presto a J. C. G. es demostrar que sus gorilas son muy capaces: «casi» son como niños. El mío (también inconfesado pero claramente inferible) es que los gorilas son mucho más limitados que los niños. A este respecto, su alternativa entre divergencias o diferencias no deja de ser un escape retórico. Porque si las divergencias les llevan por caminos en que sólo logran con dificultad o en condiciones «especiales» configurar un determinado comportamiento que en el niño surge espontáneamente pronto (y el mero hecho de su emergencia temprana habla de en favor de su transcendencia para la especie), en definitiva resultan ser deficientes con respecto a los niños. Por ejemplo, si -como es mi tesis- el gorila no capta, de entrada, el «gesto instrumental» o el gesto expresivo como algo dotado de sentido, todo un panel de comprensión manipulativa -el más fecundo en consecuencias tecnológico-culturales- queda desmantelado. El acceso al mismo a través del aprendizaje es, aparte del «detour» que supone, una réplica inacabada («funciona» a veces «como si» pero no se trata del mismo sistema funcional). Una vez más, traigo por memoria el «lenguaje» de los antropoides. Podrá aducirse que el gorila no necesita de determinadas capacidades (manipulativas o comunicativas) en su nicho ecológico, tal como hoy día lo conocemos, pero no deja de ser una divergencia que se salda con un déficit vis à vis de los humanos. No tiene sentido comparar las capacidades de dos especies sino "trasponiendo» una de ellas al nicho ecológico de a otra (aunque no sea más que a efectos de la comparación). El telón de fondo de la investigación antropoide es el comportamiento humano que ha evolucionado en otro nicho (en gran medida obra de su tecnología). Si cuando los antropoides logran esto o aquello se resalta su semejanza con los humanos y cuando aparecen claras diferencias (déficits) se habla de divergencias esto es dar pábulo a un recurso acomodaticio a las palabras. J. C. G. afirma que un enfoque basado en la idea de divergencia rechaza el esquema clásico de la Scala Naturae pero su argumento no tiene mucho fuerza porque nadie mantiene hoy un modelo «lineal » de esta última. A partir de que yo propongo que las manipulaciones en forma de reacción circular terciaria y ciertos actos comunicativos en torno a los objetos son rasgos diferenciales del desarrollo humano se sigue que los gorilas no alcanzan el nivel de intersubjetividad secundaria. ¿A partir de que J. C. G. niega mi premisa, es concluible que los gorilas alcanzan esta fase? Aquí él coincide conmigo en que no, pero por razones distintas a las mías. Pero lo que no acepto es que haya una intersubjetividad secundaria para los humanos y otra para los gorilas (la versión «hard" y la «soft» 0 , en lenguaje de ferrocarriles, «de primera» $y$ "de segunda»). Eso es crear conceptos-acordeón y toca muy de cerca a un problema epistemológico muy serio cual es el de la adecuación a los animales de ciertos conceptos acuñados para dar cuenta de fenómenos típicamente humanos. (Lo cual es una tremenda paradoja pues solo podemos hablar de fenómenos infra o extrahumanos con palabras que están genuinamente referidas a fenómenos humanos).

La constatación final es que el «hilo coductor» de J. C. G. y el mío, el alcance que damos a los mismos conceptos, difieren. Incluso me atrevería a decir que la manera como manejamos estos y los articulamos frente a los datos brutos es también distinto. En estas condiciones es difícil el acuerdo. En cualquier caso es importante proseguir la discusión aunque el debate tiene trazas de ser tan inacabable (y por las mismas razones) como la Torre de Babel. 


\section{Referencias}

CAll, J. y Alonso, C. (1980): Social learning, transmission and culture in chimpanzees. Contribution to the Joint Research (Acción Integrada) between Universidad Auto. Barcelona y University of Edinburgh.

ECKBLAD. G. (1982): Scheme Theory. Academic Press.

FOSSEY, D. (1983): Gorilas in the Mist. Hodder \& Stoughton.

HAYES, C. (1951): The Ape in our House. Harper \& Brothers.

HUNT, JMcV. (1965): Intrinsic motivation and its role in psychological development. En D.

LEVINE (Ed.) Nebraska Symposium on Motivation, Vol. 13. Un. of Nebraska Press.

KAYE, K. (1982): The Mental and Social life of Babies. The Harvester Press.

Maturana, H. y Varela, F. (1980): Autopoiesis and Cognition. Reidel Boston.

Nuttin, J. (1980): Théorie de la motivation bumaine. París. PUF.

Perinat, A. (1989): Los fundamentos psicobiológicos de la intersubjetividad. Arbor, Nov.

Tomasello, M. (1989): Chimpanzee culture? SRCD Newsletter, Winter.

WHITE, R. W. (1959): Motivation reconsidered. The concept of competence. Psychol. Rev. 66, 297-333. 